Research Article

\title{
Green Synthesis of Hydroxyethyl Cellulose-Stabilized Silver Nanoparticles
}

\author{
M. A. El-Sheikh, S. M. El-Rafie, E. S. Abdel-Halim, and M. H. El-Rafie \\ National Research Centre, Textile Research Division, El-Behooth Street, Dokki, P.O. 12311, Cairo, Egypt \\ Correspondence should be addressed to M. A. El-Sheikh; dr.manal.elsheikh@gmail.com
}

Received 28 March 2013; Revised 13 June 2013; Accepted 14 June 2013

Academic Editor: Joo Hyun Kim

Copyright (C) 2013 M. A. El-Sheikh et al. This is an open access article distributed under the Creative Commons Attribution License, which permits unrestricted use, distribution, and reproduction in any medium, provided the original work is properly cited.

\begin{abstract}
Green synthesis aims to minimize the use of unsafe reactants and maximize the efficiency of synthesis process. These could be achieved by using environmentally compassionate polymers and nontoxic chemicals. Hydroxyethyl cellulose (HEC), an ecofriendly polymer, was used as both reducing and stabilizing agents in the synthesis of stable silver nanoparticles, while silver nitrate was used as a precursor and water as a solvent. The formation of silver nanoparticles was assessed by monitoring UV-vis spectra of the silver colloidal solution. The size of the nanoparticles was measured using transmission electron microscope (TEM). Reaction kinetics was followed by measuring the absorbance of silver colloidal solution at different time intervals. Optimum reaction conditions revealed that the highest absorbance was obtained using $\mathrm{HEC}: \mathrm{AgNO}_{3}$ of $1.5: 0.17\left(\mathrm{~g} / 100 \mathrm{~cm}^{3}\right)$ at $70^{\circ} \mathrm{C}$ for $120 \mathrm{~min}$ at $\mathrm{pH} 12$. The $\mathrm{Ag}^{0}$ nanoparticles colloidal solution so obtained $(1000 \mathrm{ppm})$ were found stable in aqueous solution over a period of six months at room temperature $\left(\sim 25^{\circ} \mathrm{C}\right)$. The sizes of these nanoparticles were found in the range of $11-60 \mathrm{~nm}$ after six months of storing. FTIR spectra confirmed the interaction of both the aldehyde and $\mathrm{OH}$ groups in the synthesis and stabilization of silver nanoparticles.
\end{abstract}

\section{Introduction}

Nanotechnology is an enabling technology which deals with structures ranging from approximately 1 to $100 \mathrm{~nm}$ in at least one dimension. The nanosize results in specific physicochemical characteristics that may differ from those of the bulk substance or particles of larger size. This effect is mainly attributed to high surface area to volume ratio, which potentially results in high reactivity. Because of these specific characteristics, the use of substances in nanoform may have advantages over the use of bulk chemicals. Silver has been used since ancient times for jewelry, utensils, monetary and currency, dental alloy, photography, explosives, and so forth. Until the introduction of antibiotics, silver was also used for its antiseptic activity, specifically in the management of open wounds and burns. While an antibiotic can kill as many as a half-dozen different kinds of disease organisms, silver is known to kill 650 species of bacteria or viruses simply by coming into contact with them. Due to its antimicrobial properties, silver has also been used in medical textile applications [1-4] and incorporated in filters to purify drinking water and clean swimming pool water. Particle size has been the only limiting factor to silver's effectiveness [5].
$\mathrm{Ag} / \mathrm{Al}_{2} \mathrm{O}_{3}$ catalysts are used in the catalytic reduction of $\mathrm{NO}_{x}$ [6-8].

Increasing understanding about the environment has led to focus on "green chemistry." Although metal nanoparticles exhibited properties due to quantum size effects, their synthesis protocol creates major environmental problems [9-11]. Generally, metal nanoparticles can be prepared and stabilized by physical and chemical methods; the chemical approach, such as chemical reduction, electrochemical techniques, and photochemical reduction, is the most widely used method. Chemical reduction is the most frequently applied method for the preparation of silver nanoparticles as stable, colloidal dispersions in water or organic solvents. Commonly used reductants and solvents are sodium borohydride, citrate, ascorbate, formaldehyde, N,N-dimethylformamide, hydrazine and elemental hydrogen. These chemicals are highly reactive and cause potential environmental and biological risks [12,13]. Utilization of nontoxic chemicals, environmentally gentle solvents, and renewable materials is one of the key issues that worth important consideration in a green synthesis policy [14-16]. Biosynthesis is also an alternative green technique for synthesis of silver nanoparticles [17-21]. 
In earlier reports, natural polymers like starch [12, 22-28], chitosan [29], and cellulose [30-32] were reported to stabilize silver nanoparticles. Silver nanorods with average diameters of $20 \mathrm{~nm}$ and lengths up to $250 \mathrm{~nm}$ were successfully prepared in large scale using HEC at which silver nitrate was reduced by $\gamma$ irradiation [31].

Silver can be present in four different oxidation states: $\mathrm{Ag}^{0}, \mathrm{Ag}^{+}, \mathrm{Ag}^{2+}$, and $\mathrm{Ag}^{3+}$. The elemental silver is $\mathrm{Ag}^{0}(0$ oxidation state) and free silver ion is $\mathrm{Ag}^{+} \cdot \mathrm{Ag}^{0}$ and $\mathrm{Ag}^{+}$are the most abundant ones, while $\mathrm{Ag}^{2+}$ and $\mathrm{Ag}^{3+}$ are unstable in the aquatic environment. The reduction of silver ions $\left(\mathrm{Ag}^{+}\right)$in aqueous solution generally yields colloidal silver with particle diameters of several nanometers. Initially, the reduction of various complexes with $\mathrm{Ag}^{+}$ions leads to the formation of silver atoms $\left(\mathrm{Ag}^{0}\right)$, which is followed by agglomeration into oligomeric clusters. These clusters eventually lead to the formation of colloidal Ag particles. When the colloidal particles are much smaller than the wavelength of visible light, the solutions have a yellow color with an intense band in the 380-400 $\mathrm{nm}$ range and other less intense or smaller bands at longer wavelength in the absorption spectrum. This band is attributed to collective excitation of the electron gas in the particles, with a periodic change in electron density at the surface (surface plasmon absorption). Since the polymers prevent agglomeration and precipitation of the particles, they have been frequently employed as stabilizers in chemical synthesis of metal nanoparticles $[5,33,34]$.

Since the polymers prevent agglomeration and precipitation of the particles, they have been frequently employed as capping or stabilizing agents in chemical synthesis of metal nanoparticles. Among methods of green synthesis of silver nanoparticles, water is used as an environmentally benign solvent and polysaccharides as a capping agent, or in some cases, polysaccharides serve as both a reducing and a capping agent. Synthesis of starch-silver nanoparticles was carried out with starch as a capping agent and $\beta$-D-glucose as a reducing agent in a gently heated system. The starch in the solution mixture avoids use of relatively toxic organic solvents. In addition, the binding interactions between starch and silver nanoparticle are weak and can be reversible at higher temperatures, allowing separation of the synthesized particles. In a case of dual polysaccharide function, silver nanoparticles were synthesized by the reduction of $\mathrm{Ag}^{+}$inside of nanoscopic starch templates. The extensive network of hydrogen bonds in the templates provides surface passivation or protection against nanoparticle aggregation [23].

Cellulose and its derivatives showed unique advantages because they are the most abundant natural polysaccharide with low cost, better biodegradability, and biocompatibility. HEC is a representative derivative of cellulose with excellent water solubility and biocompatibility. HEC is manufactured by reacting alkali-treated cellulose with ethylene oxide at which a series of hydroxyethyl cellulose is produced. The manner in which ethylene oxide is added to cellulose can be described by two terms: Degree of substitution (DS) and molar substitution (MS). The degree of substitution designates the average number of hydroxyl positions on the anhydroglucose unit that has been reacted with ethylene oxide.
Since each anhydroglucose unit of the cellulose molecule has three hydroxyl groups, the maximum value for D.S. is three (Scheme 1(a)). Molar substitution is defined as the average number of ethylene oxide molecules that have reacted with each anhydroglucose unit. Once a hydroxyethyl group is attached, it can further react with additional groups in an end-to-end formation. As long as ethylene oxide is available, this reaction can continue, theoretically without limit (Scheme 1(b)). The excellent properties of HEC allow it to be used in many biotechnological, biophysical, and industrial fields. Because of the existence of abundant reactive -OH groups on the HEC chains, HEC is liable to be modified to derive new materials with improved properties $[35,36]$.

To fulfill the growing need of environmental friendly nanoparticles synthesis, this work aims to prepare environmentally friendly silver nanoparticles using HEC (a green reagent) and simple method at mild reaction conditions. Being an organic polymer bearing abundant reactive $-\mathrm{OH}$ groups on its chains, HEC can act as both reducing agent for silver nitrate and stabilizing agent for silver nanoparticles formed. Silver nitrate is used as a precursor and water as a solvent. Sodium hydroxide is used to adjust the $\mathrm{pH}$ of the reaction to $\mathrm{pH} 12$. Thus, the reaction medium only contains HEC, silver nitrate, sodium hydroxide, and distilled water. No more chemicals are used. Factors affecting the reduction and stability as well as the shape and size of the formed silver nanoparticles along with reaction mechanism are studied.

\section{Experimental}

2.1. Materials. Hydroxyethyl cellulose medium viscosity 1 (75-125 mPa.s) was purchased from FLUKA BioChemika. Silver nitrate and sodium hydroxide were of laboratory grade chemicals.

\subsection{Method}

2.2.1. Preparation of Silver Nanoparticles. Definite weight of HEC was dissolved in distilled water using heating magnetic stirrer. After complete dissolution, the $\mathrm{pH}$ of the solution was adjusted to 12 using dilute solution of sodium hydroxide. The temperature was raised gradually to reach to the desired temperature $\left(60-90^{\circ} \mathrm{C}\right)$. Certain volume of diluted silver nitrate solution was then added dropwise to the mixture keeping in mind that the total volume of the reaction mixture is $100 \mathrm{~cm}^{3}$ and silver nitrate concentration varies from 0.017 to $0.17 \mathrm{~g} / 100 \mathrm{~cm}^{3}$. The reaction mixture was kept under continuous stirring for different durations (5-180 min). After addition of silver nitrate solution, the reaction medium starts to acquire a clear yellow color which develops to brown indicating the formation of silver nanoparticles $\left(\mathrm{Ag}^{0}\right)$. After completion of the synthesis, the colloidal solution formed was checked for the presence of excess silver ions $\left(\mathrm{Ag}^{+}\right)$ using dilute $\mathrm{NaCl}$ solution. When no white precipitate is observed, this means the complete transformation of $\left(\mathrm{Ag}^{+}\right)$to silver nanoparticles $\left(\mathrm{Ag}^{0}\right)$. The development of the reaction was evaluated by withdrawing aliquots from resultant $\mathrm{Ag}^{0}$ colloidal solution at given time intervals. 


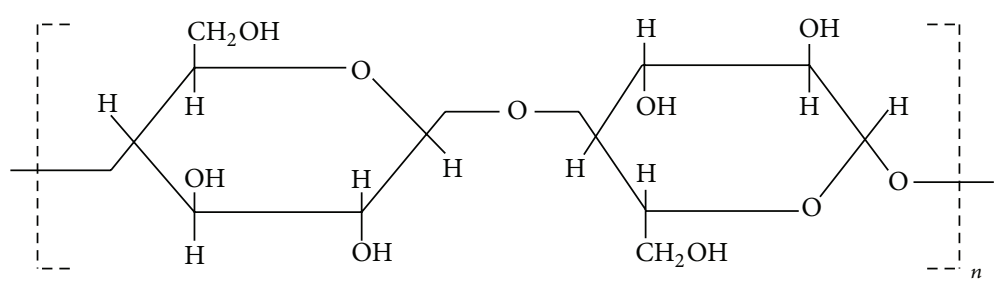

(a) Cellulose

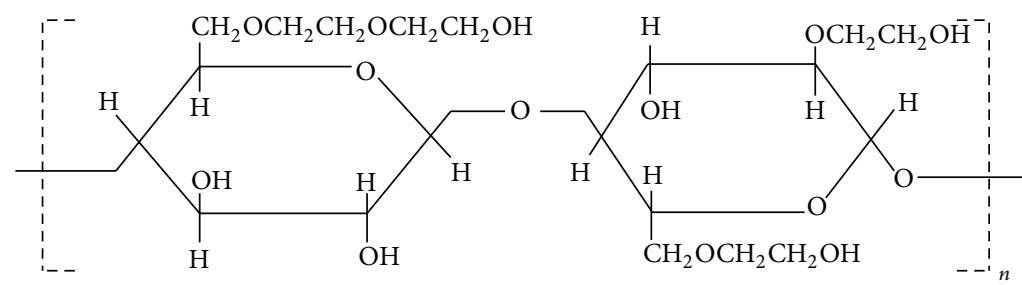

(b) HEC

Scheme 1: Structure of (a) cellulose and (b) HEC.

To obtain a solid substance of HEC-silver nanoparticles for the Fourier transform infrared (FTIR) measurements, the HEC-silver nanoparticles colloidal solution was precipitated using a mixture of absolute acetone and ethanol $(1: 1)$. After precipitation, the solution was allowed to settle down; the solvents were separated by decantation then filtration. The solid substance was air oven dried at $30^{\circ} \mathrm{C}$.

2.3. Testing and Analysis. The UV-vis spectra of silver nanoparticles were recorded using UV-2401, UV-vis spectrophotometer, Shimadzu, Japan, at wavelength range from 300 to $550 \mathrm{~nm}$. Formation of silver nanoparticles is expressed as absorbance of the colloidal solution of the samples under test. The absorbance, the broadening, and the wavelength of the band measure the intensity of the colloidal solution, that is, the conversion of silver ions to silver nanoparticles. A solution containing HEC alone was used as blank. Very concentrated silver nanoparticles samples did not show one smooth band (either sharp or broad) but showed a number of crowded sharp bands. This behavior leads to false readings. So, concentrated samples which showed this behavior were diluted " $x$ " times, and the obtained absorbance value was then multiplied by " $x$ " to obtain the actual absorbance value. The accuracy of this dilution technique was tested by comparing the absorbance readings of certain samples with moderate concentration before and after dilution. The readings were found approximately the same after multiplying by " $x$ " times of dilutions.

Transmission electron microscope was used to characterize the shape and size of the synthesized silver nanoparticles. This was done by means of a JEOL-JEM-1200 transmission electron microscope. The samples were prepared by placing a drop of the colloidal solution on a 400 mesh copper grid coated by an amorphous carbon film and evaporating the solvent in air at room temperature. The average diameter of the silver nanoparticles was determined from the diameter of
100 nanoparticles found in several chosen areas in enlarged microphotographs.

Stability of the silver nanoparticles was tested by storing samples in sealed glass bottles in dark place at room temperature $\left(\sim 25^{\circ} \mathrm{C}\right)$ for six months then recording the spectra of the solutions using UV-vis spectrophotometer as well as the shape and size using TEM.

FTIR spectra of both HEC and HEC-silver nanoparticles were obtained using Perkin-Elmer spectrum 1000 spectrophotometer. The solid samples were mixed with $\mathrm{KBr}$ forming round disk for the FTIR measurements. The FTIR spectra were scanned over a range of wave numbers from 4000 to $400\left(\mathrm{~cm}^{-1}\right)$.

\section{Results and Discussion}

3.1. Mechanism of Silver Nanoparticles Formation and Stabilization Using HEC. The reduction of transition metal salts in solution is the most widely practiced method of generating colloidal suspensions of the metals. The so-called "alcohol reduction process" is a very general process for the production of metal nanoparticles, often stabilized by organic polymers. In general, the alcohols which were useful reducing agents contained $\alpha$-hydrogen and were oxidized to the corresponding carbonyl compounds [37].

Hydroxyethyl cellulose macromolecule consists of chemically modified linear polymeric cellulose. In addition to the original alcoholic groups, new alcoholic groups are introduced as a result of the hydroxyethylation process (Scheme 1(b)). Also, in addition to original reducing aldehydic end groups in the HEC, new aldehydic end groups are generated as a result of the oxidative degradation of HEC molecule during the synthesis of silver nanoparticles under the effect of sodium hydroxide $(\mathrm{pH} 12)$ at relatively high temperature $\left(60-90^{\circ} \mathrm{C}\right)$ in the synthesis medium. While the reduction keeps on, silver nanoparticles grow gradually, and HEC will further form a stable protection layer on the 
silver nanoparticles surface. All these components with their anionic and reducing properties support the utilization of HEC as a reducing and stabilizing agent for the synthesis of silver nanoparticles.

Factors affecting the reduction and stability as well as the shape and size of the formed silver nanoparticles along with reaction mechanism are given in what follows.

\subsection{Effect of HEC Concentration and Reaction Time on the} Formation of Silver Nanoparticles. Figure 1 shows the effect of increasing HEC concentration (HEC) along with increasing reaction time on the formation of silver nanoparticles. Figures 1(a)-1(d) represent the spectra of silver nanoparticles colloidal solution after different lengths of time (5-120 min) at HEC of $0.15,0.3,0.6$, and $0.9 \mathrm{~g} / 100 \mathrm{~cm}^{3}$, respectively. At HEC of $0.15 \mathrm{~g} / 100 \mathrm{~cm}^{3}$, up to $30 \mathrm{~min}$, the bands are very broadened with little absorbance values indicating little conversion of $\mathrm{Ag}^{+}$to $\mathrm{Ag}^{0}$. After $45 \mathrm{~min}$, the broadening of the band decreases and the absorbance value increases indicating better conversion to $\mathrm{Ag}^{0}$. From 60 to $120 \mathrm{~min}$, the bands are sharp and a "bell shape" is represented with higher absorbance values. It is also clear that absorbance values at 60,90 , and $120 \mathrm{~min}$ are nearly the same showing a state of leveling off. Leveling off at 60-120 min means that no more conversion to silver nanoparticles will take place at the set of reaction conditions. At $\mathrm{HEC}$ of $0.3 \mathrm{~g} / 100 \mathrm{~cm}^{3}$, up to $15 \mathrm{~min}$, the bands are broad with little absorbance, and starting from 20 min, broadening of the bands decreases while absorbance increases. A state of leveling off is reached at 30-60 min where sharp bands with bell shapes and higher absorbance values are reached. At HEC of $0.6 \mathrm{~g} / 100 \mathrm{~cm}^{3}$, the band at $15 \mathrm{~min}$ is broad and is not representing the formation of silver nanoparticles. At 30-120, the bands are sharp, bell shaped with high absorbance which clearly expresses the formation of silver nanoparticles. At HEC of $0.9(\mathrm{w} / \mathrm{v})$, at $5 \mathrm{~min}$ the band is broad with low absorbance, while after $10 \mathrm{~min}$, the band broadening is less indicating partial conversion of silver ions to silver nanoparticles. At 15-120, the bands are sharp, bell shaped, and the absorbance is higher than after $10 \mathrm{~min}$. The absorbance at $15-120$ is nearly the same meaning that a state of leveling off that started after 15 min took place. It is clear from the previous results that by increasing the HEC concentration, the formation of silver nanoparticles increases at even lower synthesis time. To conclude, bellshaped bands with high absorbance values appeared after 60 , 30,30 , and $15 \mathrm{~min}$ for HEC $0.15,0.3,0.6$, and 0.9 , respectively. The conversion of $\mathrm{Ag}^{+}$to $\mathrm{Ag}^{0}$ as a result of increasing the concentration of HEC could be attributed to the increase in the number of reducing groups and to the enhancement in the stabilization efficiency of the formed silver nanoparticles. It should also be mentioned that the least amount of HEC in the reaction medium $\left(0.3 \mathrm{~g} / 100 \mathrm{~cm}^{3}\right)$ is enough for full reduction of the $\mathrm{Ag}^{+}$to $\mathrm{Ag}^{0}$ nanoparticles. To focus on the effect of HEC on the formation of silver nanoparticles alone while keeping the synthesis time fixed at $60 \mathrm{~min}$, a small figure was introduced (Figure 1(d)(D)). Figure 1(d)(D) shows four sharp bands with the "bell shape" at wavelength 390420 which is identical to the formation of silver nanoparticles
$[23,38]$. Although the increase in HEC favors the rate of formation of silver nanoparticles in its initial stage, and after $60 \mathrm{~min}$, the behavior is quite different. Figure $1(\mathrm{~d})(\mathrm{D})$ shows that the increase in HEC from 0.15 to 0.3 is accompanied by an increase in the absorbance. Further increase in HEC from 0.6 to 0.9 is accompanied by marginal decrement in the absorption intensity to reach its minimal value at 0.9 which could be attributed to some aggregation of the formed silver nanoparticles. However, the differences in the absorbance values among the four bands are not significant.

\subsection{Effect of Temperature and Reaction Time on the Formation} of Silver Nanoparticles. Figures 2(a)-2(d) show the effect of increasing the reaction temperature from 60 to $90^{\circ} \mathrm{C}$ after different lengths of time (5-120 min) on the synthesis of silver nanoparticles. As shown in Figure 2, performing the reaction at $60^{\circ} \mathrm{C}$ is not sufficient to synthesize silver nanoparticles, regardless of the increase in reaction time up to $120 \mathrm{~min}$. This is clear from the very broad bands with no bell shape and little absorbance. However, at $70^{\circ} \mathrm{C}$, up to $15 \mathrm{~min}$, the bands are broad with little absorbance, and starting from $20 \mathrm{~min}$, broadening of the bands decreases, while absorbance increases. A state of leveling off is reached at 30-60 min where sharp bands with bell shapes and higher absorbance values are reached. At $80^{\circ}$, at $5 \mathrm{~min}$, the band is broad with little absorbance, and at $10 \mathrm{~min}$, broadening of the band decreases, while absorbance increases. A state of leveling off is reached at 15-60 min where sharp bands with bell shapes and higher absorbance values are reached. At $90^{\circ}$, at 5-10 min, the bands are broad with little absorbance, and at $15 \mathrm{~min}$, broadening of the band sharply decreased, while absorbance increased. A state of leveling off is reached at 20-90 min where sharp bands with bell shapes and higher absorbance values are reached. To summarize, bell-shaped bands with high absorbance values did not appear at $60^{\circ} \mathrm{C}$ even after up to $120 \mathrm{~min}$ but appeared at 30,15 , and $20 \mathrm{~min}$ when reaction was performed at 70,80 , and $90^{\circ} \mathrm{C}$, respectively. This could be attributed to the favorable effect of temperature on the formation of new reducing groups from HEC by oxidative degradation of HEC in presence of sodium hydroxide. On the other hand, prolonging the reaction time $(60 \mathrm{~min})$ at a temperature higher than $70^{\circ} \mathrm{C}\left(80-90^{\circ} \mathrm{C}\right)$ is accompanied by decrease in the absorbance values as seen in Figure 2(d)(D). The decrease in the absorbance value as a result of increasing the reaction temperature could be explained in terms of the weak binding interactions between HEC and silver nanoparticles which can be reversible at higher temperatures, allowing separation of the synthesized particles [23].

\subsection{Effect of Silver Nitrate Concentration and Reaction Time} on the Formation of Silver Nanoparticles. Figures 3(a)-3(d) show the effect of increasing the concentration of $\mathrm{AgNO}_{3}$ from 0.017 to $0.0 .051 \mathrm{~g} / 100 \mathrm{~cm}^{3}$ after different durations (5-180 min) on the synthesis of silver nanoparticles. As shown in Figure 3, performing the reaction using $\mathrm{AgNO}_{3}$ of $0.017 \mathrm{~g} / 100 \mathrm{~cm}^{3}$, up to $15 \mathrm{~min}$, the bands are broad with little absorbance, and starting from $20 \mathrm{~min}$, broadening of the bands decreases, while absorbance increases. A state 


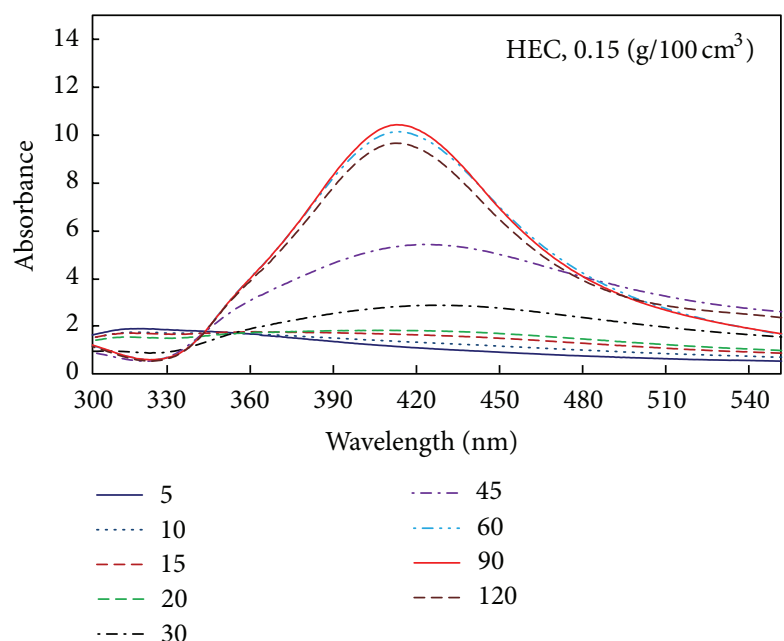

(a)

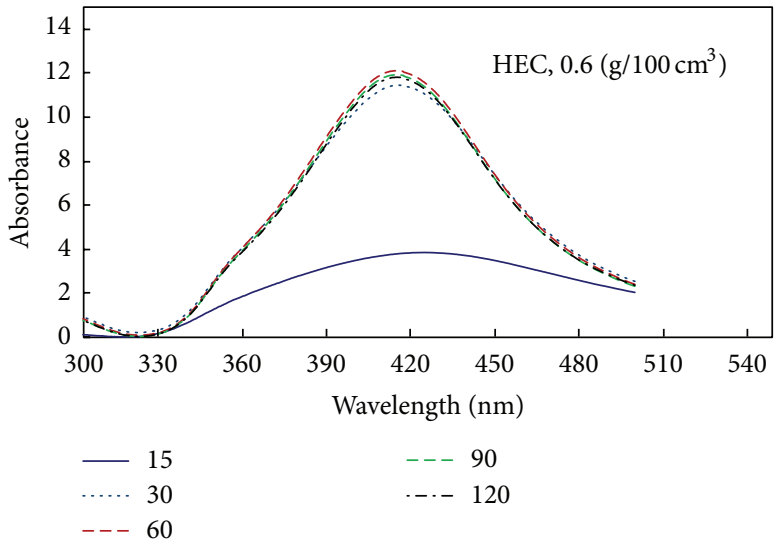

(c)

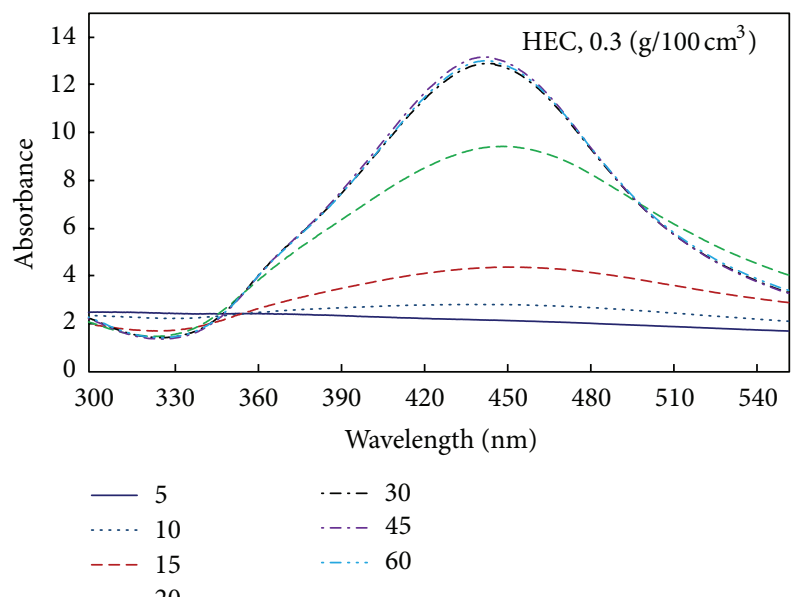

(b)

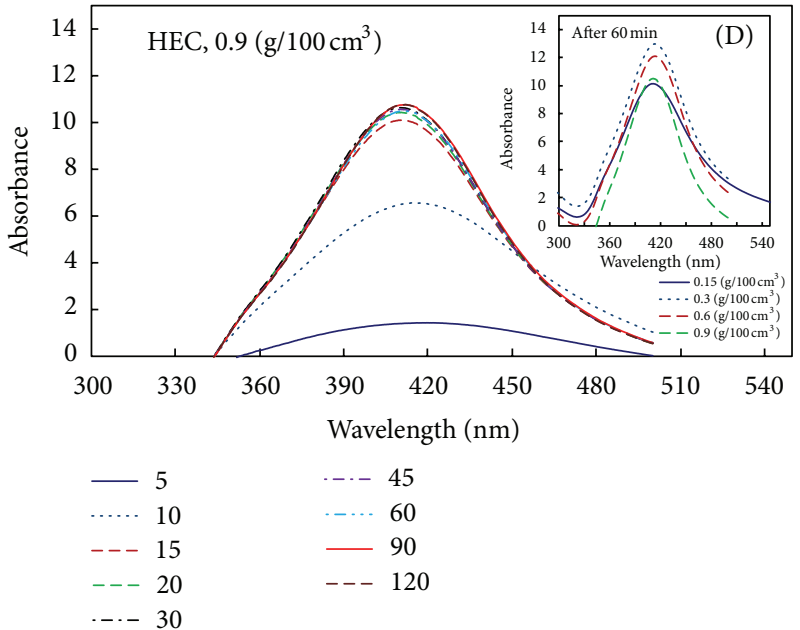

(d)

FiguRE 1: Effect of HEC concentration and reaction time on the formation of silver nanoparticles $\mathrm{HEC}, 0.15-0.9 \mathrm{~g} / 100 \mathrm{~cm}^{3}$; $\mathrm{pH}, 12$; temperature, $70^{\circ} \mathrm{C} ; \mathrm{AgNO}_{3}, 0.017 \mathrm{~g} / 100 \mathrm{~cm}^{3}$; time, 5-120 min.

of leveling off is reached at 30-60 min where bands with bell shapes and higher absorbance values are reached. At $\mathrm{AgNO}_{3}$ of $0.034 \mathrm{~g} / 100 \mathrm{~cm}^{3}$, up to $45 \mathrm{~min}$, the bands are broad with little absorbance. At $60 \mathrm{~min}$, broadening of the band decreases, while absorbance increases. The broadening continued to decrease and the absorbance to increase until it reaches its maximum at $120 \mathrm{~min}$ where bands with bell shapes and higher absorbance values are reached. At $\mathrm{AgNO}_{3}$ of $0.051 \mathrm{~g} / 100 \mathrm{~cm}^{3}$, up to $60 \mathrm{~min}$, the bands are broad with little absorbance. From 90 to $120 \mathrm{~min}$, broadening of the band slightly decreased, while absorbance increased. The broadening continued to decrease and absorbance to increase until it reaches its maximum at 180 min where a band with bell shape and higher absorbance values is reached. To conclude, bell-shaped bands with high absorbance values appeared at 30,60 , and $90 \mathrm{~min}$ when reaction was performed using $\mathrm{AgNO}_{3}$ of $0.017,0.034$, and $0.051 \mathrm{~g} / 100 \mathrm{~cm}^{3}$, respectively. Figure 3(d) which shows the effect of increasing $\mathrm{AgNO}_{3}$ on the absorbance of the colloidal solution of silver nanoparticles at $60 \mathrm{~min}$ is in agreement with these findings. It seems from the previous results that the amount of reducing groups in HEC is only sufficient to bring silver nanoparticles when silver nitrate concentration is in the range of $0.017 \mathrm{~g} / 100 \mathrm{~cm}^{3}$. More reducing groups are required to guarantee the conversion of $\mathrm{Ag}^{+}$to $\mathrm{Ag}^{0}$ when $\mathrm{AgNO}_{3}$ is to be increased to more than $0.017 \mathrm{~g} / 100 \mathrm{~cm}^{3}$. This could be achieved by increasing both $\mathrm{HEC}$ and $\mathrm{AgNO}_{3}$ together in a parallel way.

3.5. Effect of Increasing Both $\mathrm{HEC}$ and $\mathrm{AgNO}_{3}$ Concentrations Together and Reaction Time on the Formation of Silver Nanoparticles. Figures 4(a)-4(d) show the effect of increasing both $\mathrm{HEC}$ and $\mathrm{AgNO}_{3}$ concentrations together $\left(0.6: 0.068,0.9: 0.102\right.$, and $\left.1.5: 0.17\left(\mathrm{~g} / 100 \mathrm{~cm}^{3}\right)\right)$ and reaction time $(5: 120 \mathrm{~min})$ on the formation of silver nanoparticles. As shown in Figure 4, performing the reaction using HEC: $\mathrm{AgNO}_{3}$ of $0.6: 0.068$ brings bell-shaped bands with high absorbance regardless of the reaction time. Figure 4 

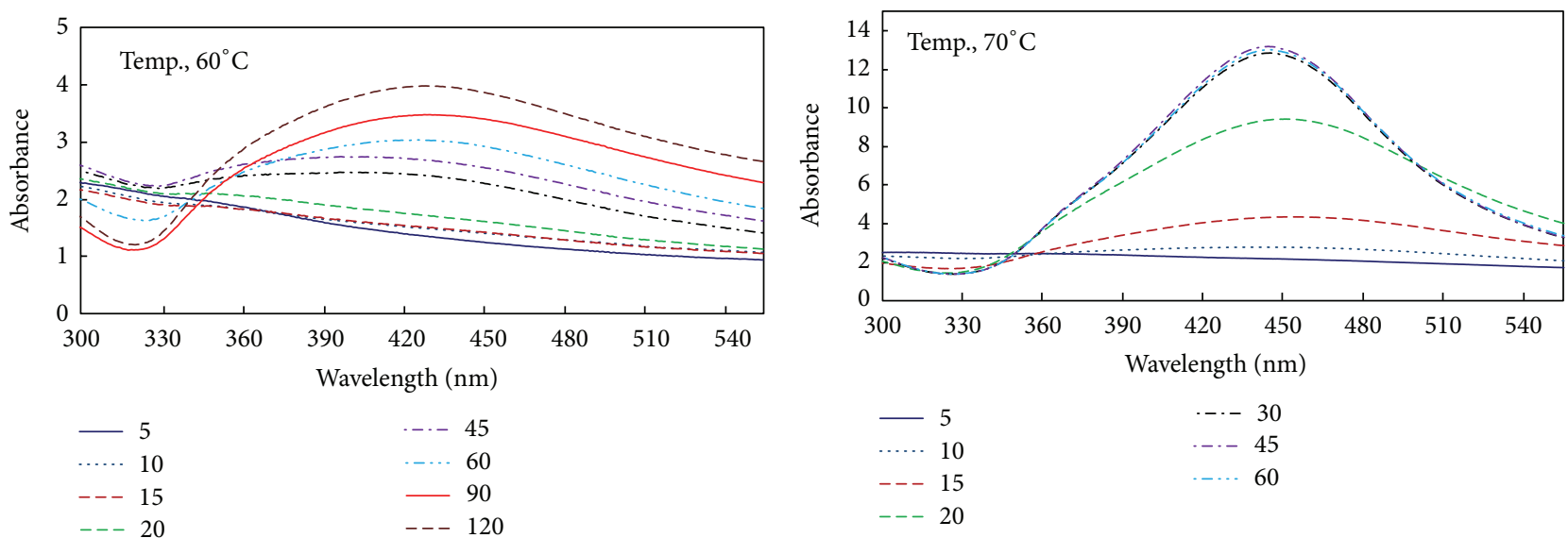

(a)

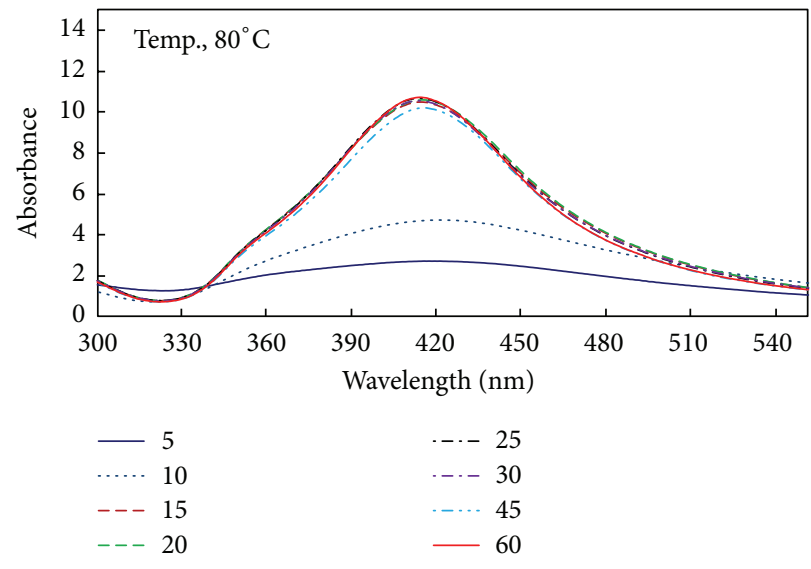

(c)

(b)

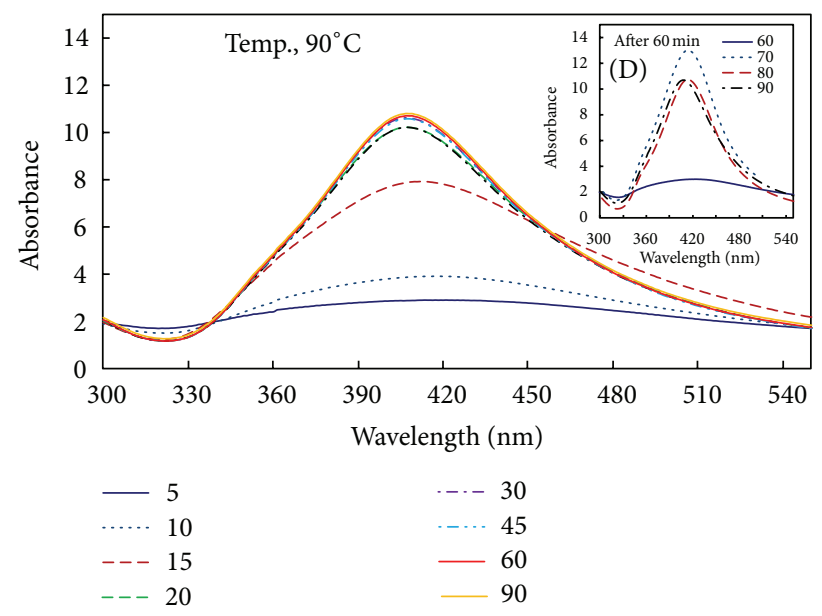

(d)

FIGURE 2: Effect of temperature and reaction time on the formation of silver nanoparticles HEC, $0.3 \mathrm{~g} / 100 \mathrm{~cm}^{3} ; \mathrm{pH}, 12$; temperature, $60-90^{\circ} \mathrm{C}$; $\mathrm{AgNO}_{3}, 0.017 \mathrm{~g} / 100 \mathrm{~cm}^{3}$; time, 5-120 min.

also shows that the absorbance increases by increasing the reaction time from 15 to $30 \mathrm{~min}$ after which it remains constant, and a state of leveling off is reached from 30 to $120 \mathrm{~min}$. By using $\mathrm{HEC}: \mathrm{AgNO}_{3}$ of $0.9: 0.102$ at $15 \mathrm{~min}$ the band is broad with little absorbance. From 90 to $120 \mathrm{~min}$, broadening of the band sharply decreased, while the absorbance sharply increased with appearance of clear bellshaped band. Using HEC: $\mathrm{AgNO}_{3}$ of $1.5: 0.17\left(\mathrm{~g} / 100 \mathrm{~cm}^{3}\right)$, at 15-30 $\mathrm{min}$, the bands are broad with little absorbance. From 60 to $120 \mathrm{~min}$, broadening of the band sharply decreased while the absorbance sharply increased with appearance of clear bell-shaped bands. The absorbance reaches its highest value at $120 \mathrm{~min}$. To conclude, bell-shaped bands with high absorbance values appeared at 15, 30, and $60 \mathrm{~min}$ when reaction was performed using $\mathrm{HEC}: \mathrm{AgNO}_{3}$ of $0.6: 0.068$, $0.9: 0.102$, and $1.5: 0.17\left(\mathrm{~g} / 100 \mathrm{~cm}^{3}\right)$, respectively. By making reaction time constant $(60 \mathrm{~min})$, as shown in Figure $4(\mathrm{~d})$, the increase in both $\mathrm{HEC}: \mathrm{AgNO}_{3}$ is accompanied by an increase in the absorbance. Results of Figure 3 and results of Figure 4 assure that there must be parallel increase in both $\mathrm{HEC}$ and $\mathrm{AgNO}_{3}$ in order to obtain high yield of silver nanoparticles where the amount of reducing groups in HEC must be sufficient to reduce all $\mathrm{Ag}^{+}$in the solution to $\mathrm{Ag}^{0}$. More concentrations of $\mathrm{AgNO}_{3}$ must be accompanied by parallel increase in HEC concentration.

3.6. Stability of Silver Nanoparticles after Six Months. Stability of silver nanoparticles was tested by measuring the absorbance of the colloidal solution after synthesis (1) and after six months of storing (2). Figure 5 shows absorbance of the colloidal solution of samples with different concentrations of both HEC and silver nitrate (HEC: $\mathrm{AgNO}_{3}, 0.6: 0.068$, $0.9: 0.102$, and $1.5: 0.17)\left(\mathrm{g} / 100 \mathrm{~cm}^{3}\right)$ at identical reaction conditions. As seen in Figure 5, the absorbance curve and the absorbance maxima of each pair of samples, namely, $0.6: 0.068$ (1) and $0.6: 0.068$ (2); $0.9: 0.102$ (1) and $0.9: 0.102$ (2), are identical showing that the silver nanoparticles are stable at the storing conditions. The pair of samples $1.5: 0.17$ (1) and 1.5:0.17 (2) showed even higher absorbance after storing for six months. The increase of the absorbance of the colloidal solution of $1.5: 0.17$ (2) after such long period 


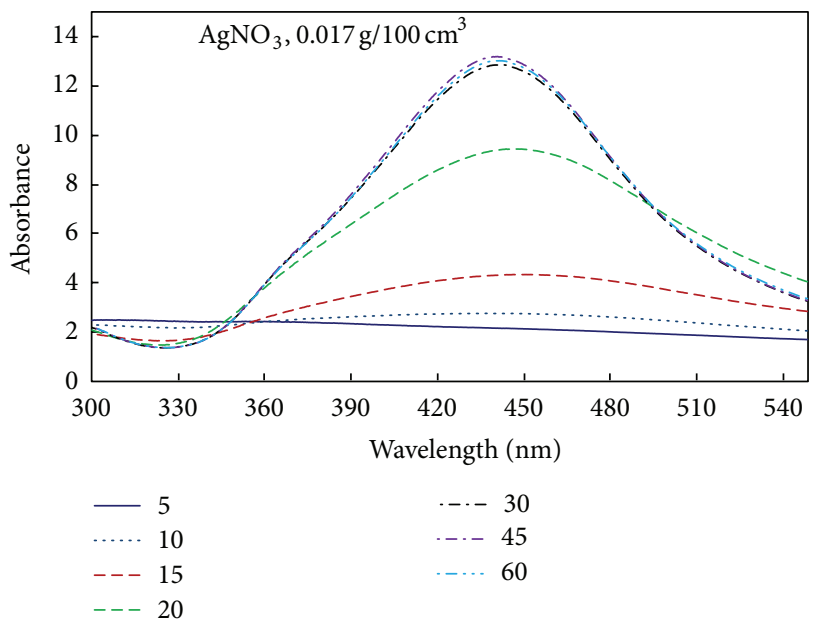

(a)

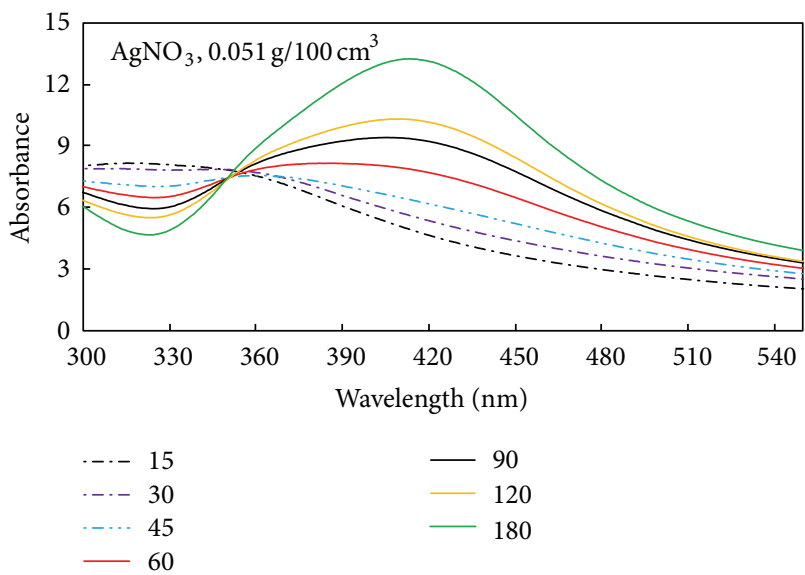

(c)

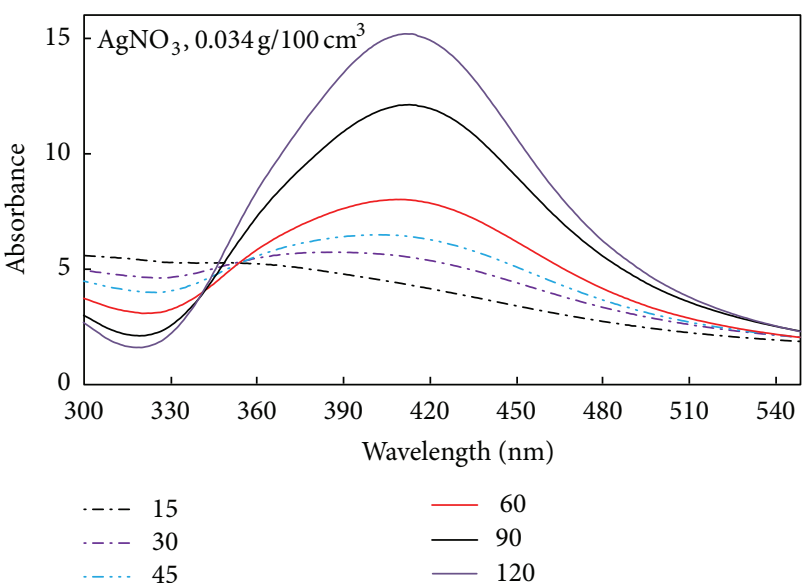

(b)

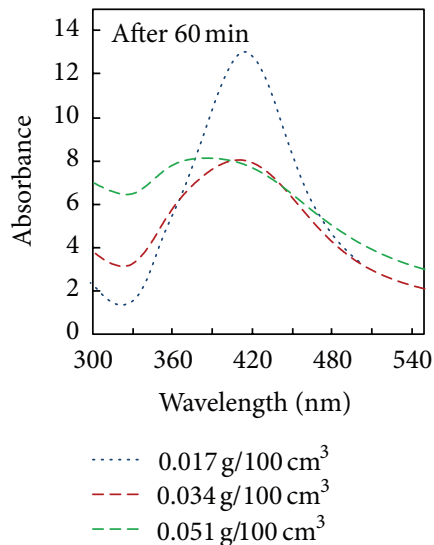

(d)

FIGURE 3: Effect of $\mathrm{AgNO}_{3}$ concentration and reaction time on the formation of silver nanoparticles $\mathrm{HEC}, 0.3 \mathrm{~g} / 100 \mathrm{~cm}^{3}$; $\mathrm{pH}, 12$; temperature, $70^{\circ} \mathrm{C} ; \mathrm{AgNO}_{3}, 0.017-0.051 \mathrm{~g} / 100 \mathrm{~cm}^{3}$; time, 5-180 min.

of storing means that the reaction was continued at room temperature until all silver irons are converted to silver nanoparticles followed by stability of the colloidal solution.

\subsection{TEM Micrograph and Particle Size Distribution Histogram} of Silver Nanoparticles. Figure 6 shows TEM micrograph and particle size distribution histogram of silver nanoparticles synthesized using different $\mathrm{HEC}: \mathrm{AgNO}_{3}\left(\mathrm{~g} / 100 \mathrm{~cm}^{3}\right)$ : (a) $0.6: 0.068$, (b) $0.9: 0.102$, and (c) $1.5: 0.17$ after six months of storing. The three micrographs show that silver nanoparticles have spherical shape morphology, fine dispersion, and mean particle sizes between 11 and 55, 6 and 55, and 11 and $60 \mathrm{~nm}$ for HEC: $\mathrm{AgNO}_{3}$ 0.6 : 0.068, 0.9 : 0.102, and $1.5: 0.17\left(\mathrm{~g} / 100 \mathrm{~cm}^{3}\right)$ respectively. Highest count\% between 26 and 30, 31 and $35 \%$ for $\mathrm{HEC}: \mathrm{AgNO}_{3}$ 0.6:0.068, 0.9:0.102 (g/100 cm $\left.{ }^{3}\right)$, respectively and $11-20$ and $31-35 \%$ for $\mathrm{HEC}: \mathrm{AgNO}_{3} 1.5: 0.17$ $\left(\mathrm{g} / 100 \mathrm{~cm}^{3}\right)$. It is clear that the increase in the $\mathrm{HEC}: \mathrm{AgNO}_{3}$ has no effect on the particle size and shape. It is worth to mention that even after six months of storing, the particle size is generally in the range of 6-60 $\mathrm{nm}$.
3.8. FTIR Spectra of HEC and HEC-Silver Nanoparticles. $\mathrm{HEC}$ is an organic polymer bearing abundant reactive $\mathrm{OH}$ groups and some aldehyde groups on its chains. High reaction temperature $\left(70^{\circ} \mathrm{C}\right)$ favors the formation of new reducing aldehyde groups by oxidative degradation of HEC in presence of sodium hydroxide ( $\mathrm{pH} 12)$. The interaction of both the aldehyde and hydroxyl groups in the reduction of silver ions and stabilization of silver nanoparticles obtained was confirmed by FTIR spectra of HEC-silver nanoparticles compared with HEC.

Figure 7 shows the FTIR spectra of both HEC and HEC-silver nanoparticles. The FTIR spectra of HEC show bands at 3410, 2928, 1644, and $1060 \mathrm{~cm}^{-1}$ characteristic for hydrogen stretching of bonded $\mathrm{O}-\mathrm{H}$, hydrogen stretching of $\mathrm{C}-\mathrm{H}$, double bond stretching of carbonyl, carboxyl and carboxyl salt, and hydrogen bending of $\mathrm{O}-\mathrm{H}$, respectively, with corresponding intensities of 57, 59, 67, and 56\% $T$, respectively. Same characteristic bands for HEC-silver nanoparticles appeared at 3426, 2928, 1647, and $1060 \mathrm{~cm}^{-1}$ at either the same frequencies $\left(2928\right.$ and $\left.1060 \mathrm{~cm}^{-1}\right)$ or with 


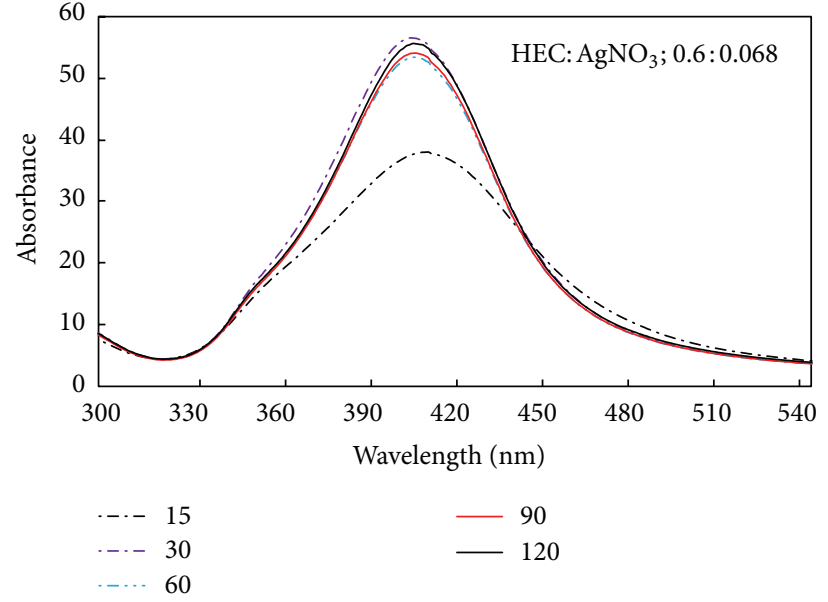

(a)

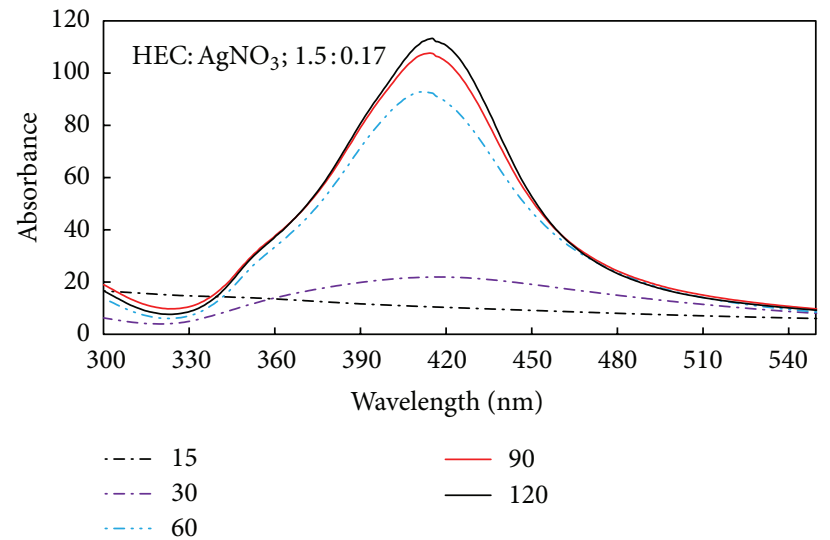

(c)

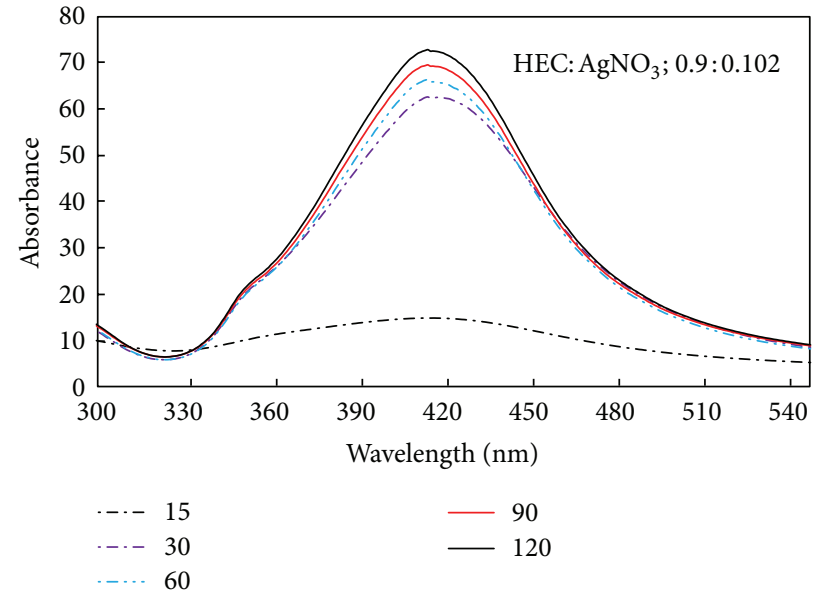

(b)

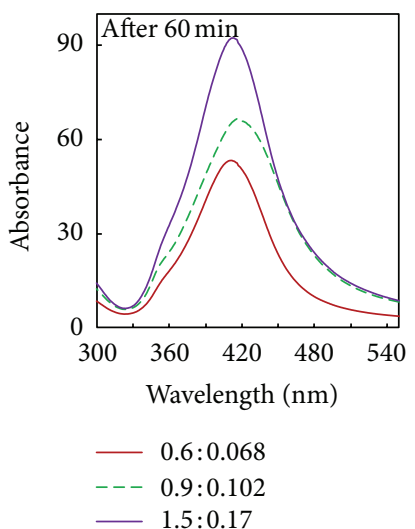

(d)

FIGURE 4: Effect of the ratio between $\mathrm{HEC}$ and $\mathrm{AgNO}_{3}$ concentration and reaction time on the formation of silver nanoparticles $\mathrm{HEC}_{\mathrm{AgNO}}$, 0.6-1.5: $0.068: 0.17\left(\mathrm{~g} / 100 \mathrm{~cm}^{3}\right) ; \mathrm{pH}, 12$; temperature, $70^{\circ} \mathrm{C}$; time, $15-120 \mathrm{~min}$.

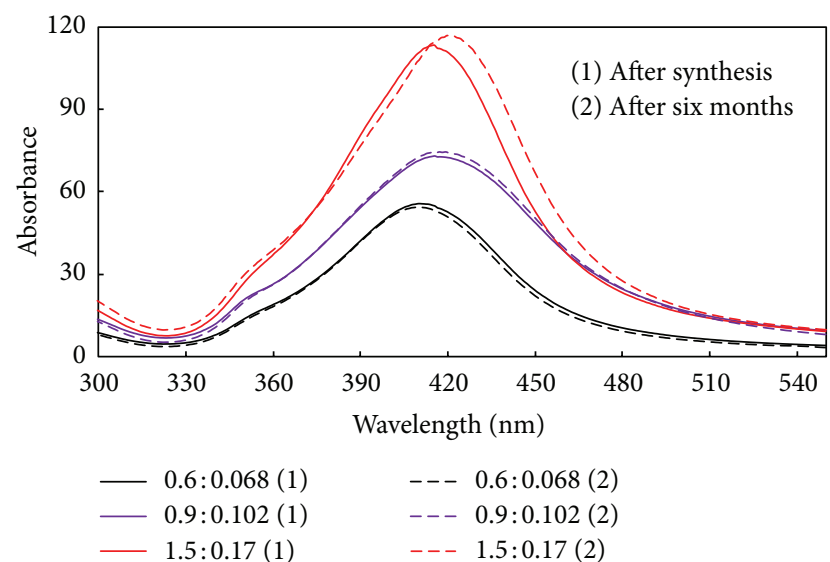

FIGURE 5: Stability of silver nanoparticles after six months. HEC : $\mathrm{AgNO}_{3}, 0.6-1.5: 0.068: 0.17\left(\mathrm{~g} / 100 \mathrm{~cm}^{3}\right) ; \mathrm{pH}, 12$; temperature, $70^{\circ} \mathrm{C}$; time, $60 \mathrm{~min}$, after six months of synthesis.

little shift to higher frequencies ( 3426 and $1647 \mathrm{~cm}^{-1}$ ). The strengths of the bands of HEC-silver nanoparticles $(86,90,87$, and $84 \% T$, resp.) are completely different from HEC. These observations confirm the interaction of both the aldehyde and the $\mathrm{OH}$ groups in the synthesis and stabilization of silver nanoparticles.

\section{Conclusions}

Green synthesis of silver nanoparticles was performed using HEC as both reducing and stabilizing agent, silver nitrate as precursor, water as solvent, and sodium hydroxide to bring alkaline reaction medium of $\mathrm{pH} 12$. At low reaction times, the increase in [HEC] favors the rate of formation of silver nanoparticles, while longer durations are not favorable. This observation was repeated in studying other parameters; that is, it is not recommended to extend reaction for more duration after synthesis is completed because aggregations of silver nanoparticles can take place. Low reaction temperature $\left(60^{\circ} \mathrm{C}\right)$ and high reaction temperatures $\left(80-90^{\circ} \mathrm{C}\right)$ have adverse effect on the synthesis of silver nanoparticles, while $70^{\circ} \mathrm{C}$ is optimal. Increasing silver nitrate concentration should be accompanied by increasing HEC concentration in a parallel way to guarantee complete conversion of $\mathrm{Ag}^{+}$to 

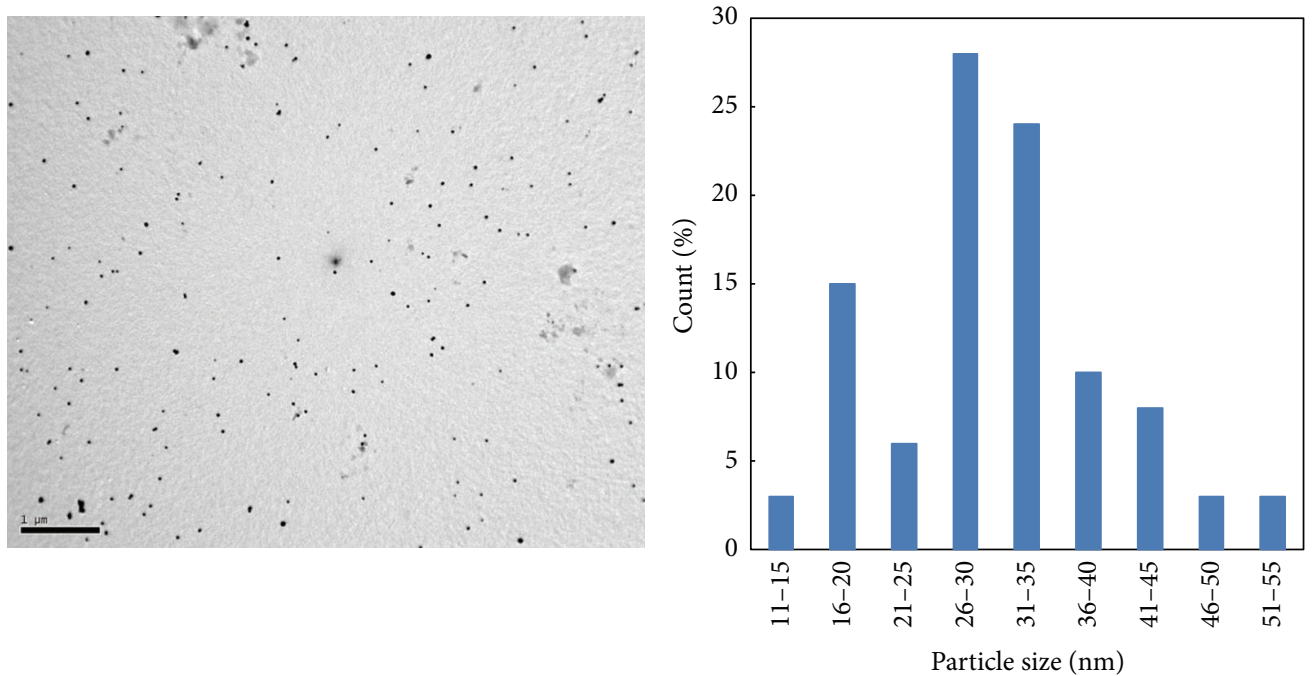

(a)
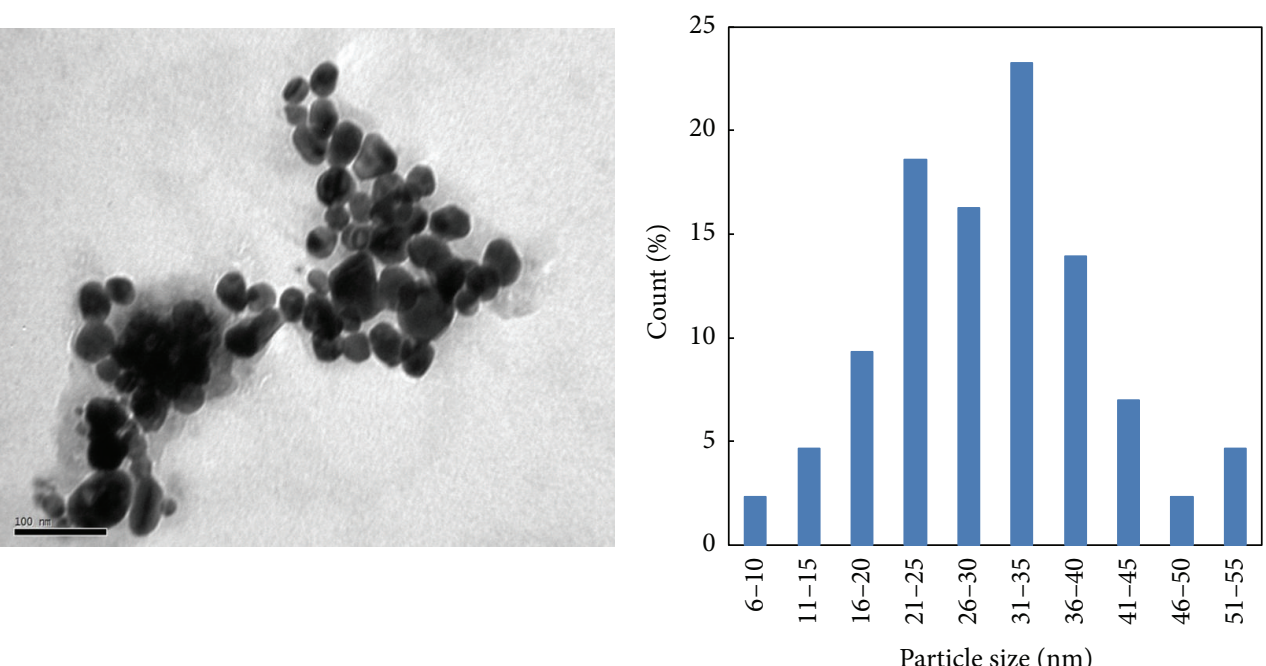

(b)
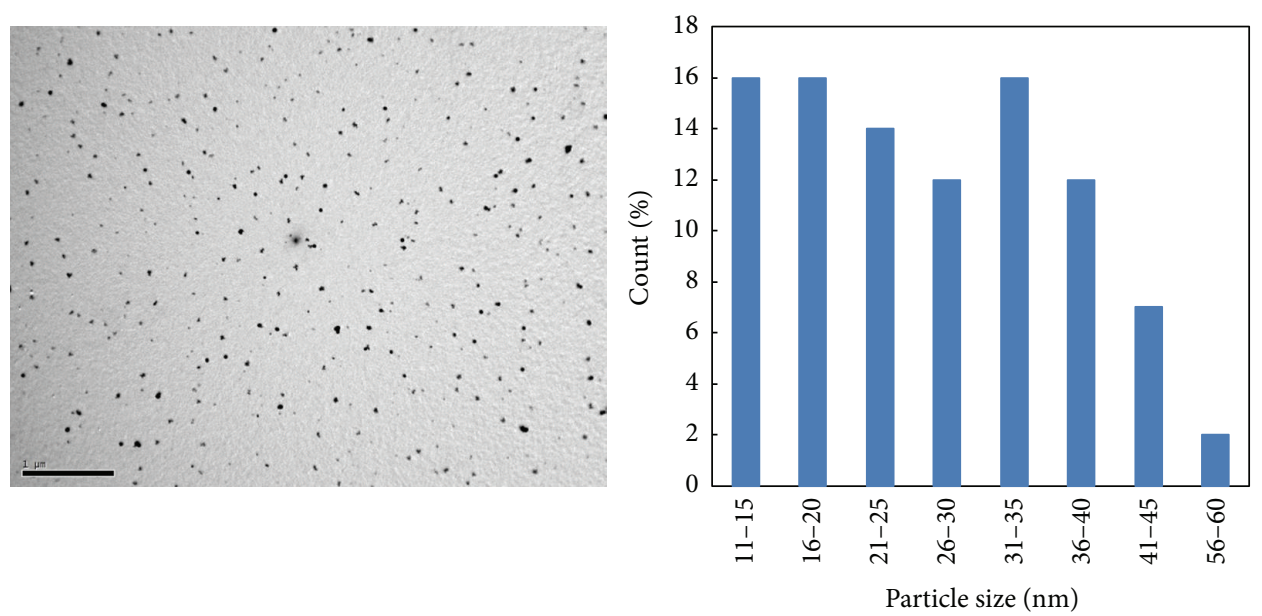

(c)

FIGURE 6: TEM micrograph and particle size distribution histogram of silver nanoparticles synthesized using different $\mathrm{HEC}_{\mathrm{AgNO}} \mathrm{AgN}_{3}$ $\left(\mathrm{g} / 100 \mathrm{~cm}^{3}\right)$ : (a) $0.6: 0.068$, (b) $0.9: 0.102$, and (c) $1.5: 0.17 \mathrm{pH}, 12$; temperature, $70^{\circ} \mathrm{C}$; time, six months. 


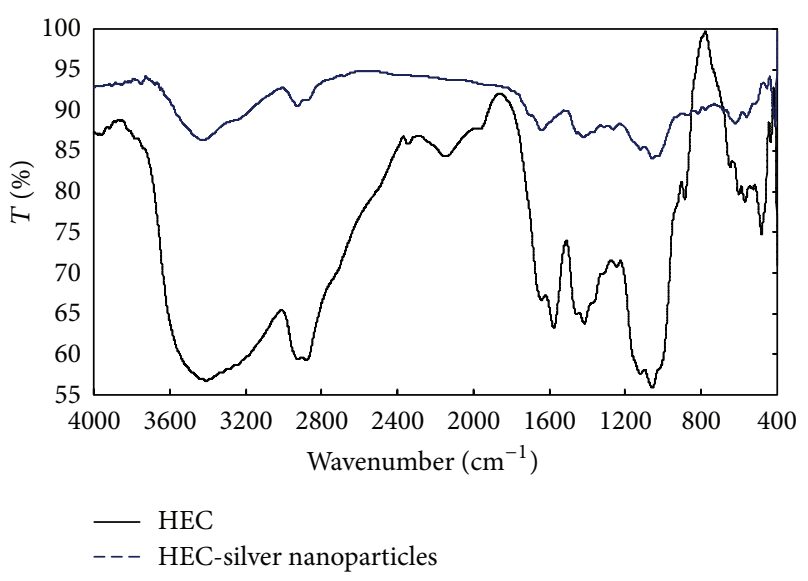

FIgURE 7: FTIR of HEC and HEC-silver nanoparticles HEC: $\mathrm{AgNO}_{3}, \quad 0.6: 0.068\left(\mathrm{~g} / 100 \mathrm{~cm}^{3}\right) ; \mathrm{pH}, 12$; temperature, $70^{\circ} \mathrm{C}$; time, $60 \mathrm{~min}$. The FTIR spectra of HEC-silver nanoparticles were obtained using the solid form of the substance.

$\mathrm{Ag}^{0}$ if we are after high yield of silver nanoparticles. Optimal reaction conditions that yield the highest absorbance values were $\mathrm{HEC}: \mathrm{AgNO}_{3}$ of $1.5: 0.17\left(\mathrm{~g} / 100 \mathrm{~cm}^{3}\right), 70^{\circ} \mathrm{C}$ for $120 \mathrm{~min}$ at $\mathrm{pH}$ 12. The nanoparticles so obtained were found stable in aqueous solution over a period of six months at room temperature $\left(\sim 25^{\circ} \mathrm{C}\right)$. The size of these nanoparticles was found in the range of $11-60 \mathrm{~nm}$ after six months of storing. FTIR spectra confirm the interaction of both the aldehyde and $\mathrm{OH}$ groups in the synthesis and stabilization of silver nanoparticles.

\section{References}

[1] A. I. Wasif and S. K. Laga, "Use of nano silver as an antimicrobial agent for cotton," Autex Research Journal, vol. 9, no. 1, pp. 5-13, 2009.

[2] D. Tessier, I. Radu, and M. Filteau, "Antimicrobial fabrics coated with nano-sized silver salt crystals," in Proceedings of the NSTI Nanotechnology Conference and Trade Show-NSTI Nanotech, M. Laudon and B. Romanowicz, Eds., pp. 762-764, Anaheim, Calif, USA, May 2005.

[3] M. Gouda, "Nano-zirconium oxide and nano-silver oxide/cotton gauze fabrics for antimicrobial and wound healing acceleration," Journal of Industrial Textiles, vol. 41, no. 3, pp. 222-240, 2012.

[4] M. H. El-Rafie, A. A. Mohamed, T. I. Shaheen, and A. Hebeish, "Antimicrobial effect of silver nanoparticles produced by fungal process on cotton fabrics," Carbohydrate Polymers, vol. 80, no. 3, pp. 779-782, 2010.

[5] S. W. P. Wijnhoven, W. J. G. M. Peijnenburg, C. A. Herberts et al., "Nano-silver-a review of available data and knowledge gaps in human and environmental risk assessment," Nanotoxicology, vol. 3, no. 2, pp. 109-138, 2009.

[6] J. P. Breen, R. Burch, C. Hardacre, and C. J. Hill, "Structural investigation of the promotional effect of hydrogen during the selective catalytic reduction of $\mathrm{NO}_{x}$ with hydrocarbons over $\mathrm{Ag} / \mathrm{Al}_{2} \mathrm{O}_{3}$ catalysts," Journal of Physical Chemistry B, vol. 109, no. 11, pp. 4805-4807, 2005.
[7] K. Arve, F. Klingstedt, K. Eränen et al., "Analysis of the state and size of silver on alumina in effective removal of $\mathrm{NO}_{x}$ from oxygen rieh exhaust gas," Journal of Nanoscience and Nanotechnology, vol. 6, no. 4, pp. 1076-1083, 2006.

[8] A. Musi, P. Massiani, D. Brouri, J.-M. Trichard, and P. Da Costa, "On the characterisation of silver species for SCR of $\mathrm{NO}_{x}$ with ethanol," Catalysis Letters, vol. 128, no. 1-2, pp. 25-30, 2009.

[9] A. Henglein, "Small-particle research: physicochemical properties of extremely small colloidal metal and semiconductor particles," Chemical Reviews, vol. 89, no. 8, pp. 1861-1873, 1989.

[10] L. N. Lewis, "Chemical catalysis by colloids and clusters," Chemical Reviews, vol. 93, no. 8, pp. 2693-2730, 1993.

[11] A. P. Alivisatos, "Semiconductor clusters, nanocrystals, and quantum dots," Science, vol. 271, no. 5251, pp. 933-937, 1996.

[12] N. Vigneshwaran, R. P. Nachane, R. H. Balasubramanya, and P. V. Varadarajan, "A novel one-pot "green" synthesis of stable silver nanoparticles using soluble starch," Carbohydrate Research, vol. 341, no. 12, pp. 2012-2018, 2006.

[13] P. Dallas, V. K. Sharma, and R. Zboril, "Silver polymeric nanocomposites as advanced antimicrobial agents: classification, synthetic paths, applications, and perspectives," Advances in Colloid and Interface Science, vol. 166, no. 1-2, pp. 119-135, 2011.

[14] P. Raveendran, J. Fu, and S. L. Wallen, "Completely "Green" synthesis and stabilization of metal nanoparticles," Journal of the American Chemical Society, vol. 125, no. 46, pp. 13940-13941, 2003.

[15] J. M. DeSimone, "Practical approaches to green solvents," Science, vol. 297, no. 5582, pp. 799-803, 2002.

[16] M. Poliakoff and P. Anastas, "A principled stance," Nature, vol. 413, no. 6853, p. 257, 2001.

[17] P. Vankar and D. Shukla, "Biosynthesis of silver nanoparticles using lemon leaves extract and its application for antimicrobial finish on fabric," Applied Nanoscience, vol. 2, no. 2, pp. 163-168, 2012.

[18] Z. Sadowski, "Biosynthesis and application of silver and gold nanoparticles," in Silver Nanoparticles, D. P. Perez, Ed., pp. 257276, In Tech, 2010.

[19] S. Kaviya, J. Santhanalakshmi, and B. Viswanathan, "Biosynthesis of silver nano-flakes by Crossandra infundibuliformis leaf extract," Materials Letters, vol. 67, no. 1, pp. 64-66, 2012.

[20] A. Saxena, R. M. Tripathi, F. Zafar, and P. Singh, "Green synthesis of silver nanoparticles using aqueous solution of Ficus benghalensis leaf extract and characterization of their antibacterial activity," Materials Letters, vol. 67, no. 1, pp. 91-94, 2012.

[21] M. H. El-Rafie, T. I. Shaheen, A. A. Mohamed, and A. Hebeish, "Bio-synthesis and applications of silver nanoparticles onto cotton fabrics," Carbohydrate Polymers, vol. 90, no. 2, pp. 915920, 2012.

[22] M. Z. Kassaee, A. Akhavan, N. Sheikh, and R. Beteshobabrud, " $\gamma$-Ray synthesis of starch-stabilized silver nanoparticles with antibacterial activities," Radiation Physics and Chemistry, vol. 77, no. 9, pp. 1074-1078, 2008.

[23] V. K. Sharma, R. A. Yngard, and Y. Lin, "Silver nanoparticles: green synthesis and their antimicrobial activities," Advances in Colloid and Interface Science, vol. 145, no. 1-2, pp. 83-96, 2009.

[24] M. Valodkar, A. Bhadoria, J. Pohnerkar, M. Mohan, and S. Thakore, "Morphology and antibacterial activity of carbohydrate-stabilized silver nanoparticles," Carbohydrate Research, vol. 345, no. 12, pp. 1767-1773, 2010. 
[25] A. Hebeish, M. E. El-Naggar, M. M. G. Fouda, M. A. Ramadan, S. S. Al-Deyab, and M. H. El-Rafie, "Highly effective antibacterial textiles containing green synthesized silver nanoparticles," Carbohydrate Polymers, vol. 86, no. 2, pp. 936-940, 2011.

[26] M. H. El-Rafie, M. E. El-Naggar, M. A. Ramadan, M. M. G. Fouda, S. S. Al-Deyab, and A. Hebeish, "Environmental synthesis of silver nanoparticles using hydroxypropyl starch and their characterization," Carbohydrate Polymers, vol. 86, no. 2, pp. 630-635, 2011.

[27] V. Djoković, R. Krsmanović, D. K. Božanić et al., "Adsorption of sulfur onto a surface of silver nanoparticles stabilized with sago starch biopolymer," Colloids and Surfaces B, vol. 73, no. 1, pp. 30-35, 2009.

[28] P. Raveendran, J. Fu, and S. L. Wallen, "A simple and "green" method for the synthesis of $\mathrm{Au}, \mathrm{Ag}$, and $\mathrm{Au}-\mathrm{Ag}$ alloy nanoparticles," Green Chemistry, vol. 8, no. 1, pp. 34-38, 2006.

[29] H. Huang, Q. Yuan, and X. Yang, "Preparation and characterization of metal-chitosan nanocomposites," Colloids and Surfaces $B$, vol. 39, no. 1-2, pp. 31-37, 2004.

[30] J. Chen, J. Wang, X. Zhang, and Y. Jin, "Microwave-assisted green synthesis of silver nanoparticles by carboxymethyl cellulose sodium and silver nitrate," Materials Chemistry and Physics, vol. 108, no. 2-3, pp. 421-424, 2008.

[31] W. Liu, Z. Zhang, H. Liu, W. He, X. Ge, and M. Wang, "Silver nanorods using HEC as a template by $\gamma$-irradiation technique and absorption dose that changed their nanosize and morphology," Materials Letters, vol. 61, no. 8-9, pp. 1801-1804, 2007.

[32] A. A. Hebeish, M. H. El-Rafie, F. A. Abdel-Mohdy, E. S. AbdelHalim, and H. E. Emam, "Carboxymethyl cellulose for green synthesis and stabilization of silver nanoparticles," Carbohydrate Polymers, vol. 82, no. 3, pp. 933-941, 2010.

[33] N. Singh and P. K. Khanna, "In situ synthesis of silver nanoparticles in polymethylmethacrylate," Materials Chemistry and Physics, vol. 104, no. 2-3, pp. 367-372, 2007.

[34] D. V. Goia, "Preparation and formation mechanisms of uniform metallic particles in homogeneous solutions," Journal of Materials Chemistry, vol. 14, no. 4, pp. 451-458, 2004.

[35] W. Wang, J. Wang, Y. Kang, and A. Wang, "Synthesis, swelling and responsive properties of a new composite hydrogel based on hydroxyethyl cellulose and medicinal stone," Composites $B$, vol. 42, no. 4, pp. 809-818, 2011.

[36] J. Reuben, "Description and analysis of hydroxyethyl cellulose," Macromolecules, vol. 17, no. 2, pp. 156-161, 1984.

[37] J. S. Bradley, G. Schmid, D. V. Talapin, E. V. Shevchenko, and H. Weller, "Syntheses and characterizations: 3.2 synthesis of metal nanoparticles," in Nanoparticles From Theory to Application, pp. 185-238, Wiley-VCH Verlag GmbH \& Co. KGaA, 2005.

[38] E. S. Abdel-Halim, M. H. El-Rafie, and S. S. Al-Deyab, "Polyacrylamide/guar gum graft copolymer for preparation of silver nanoparticles," Carbohydrate Polymers, vol. 85, no. 3, pp. 692697, 2011. 

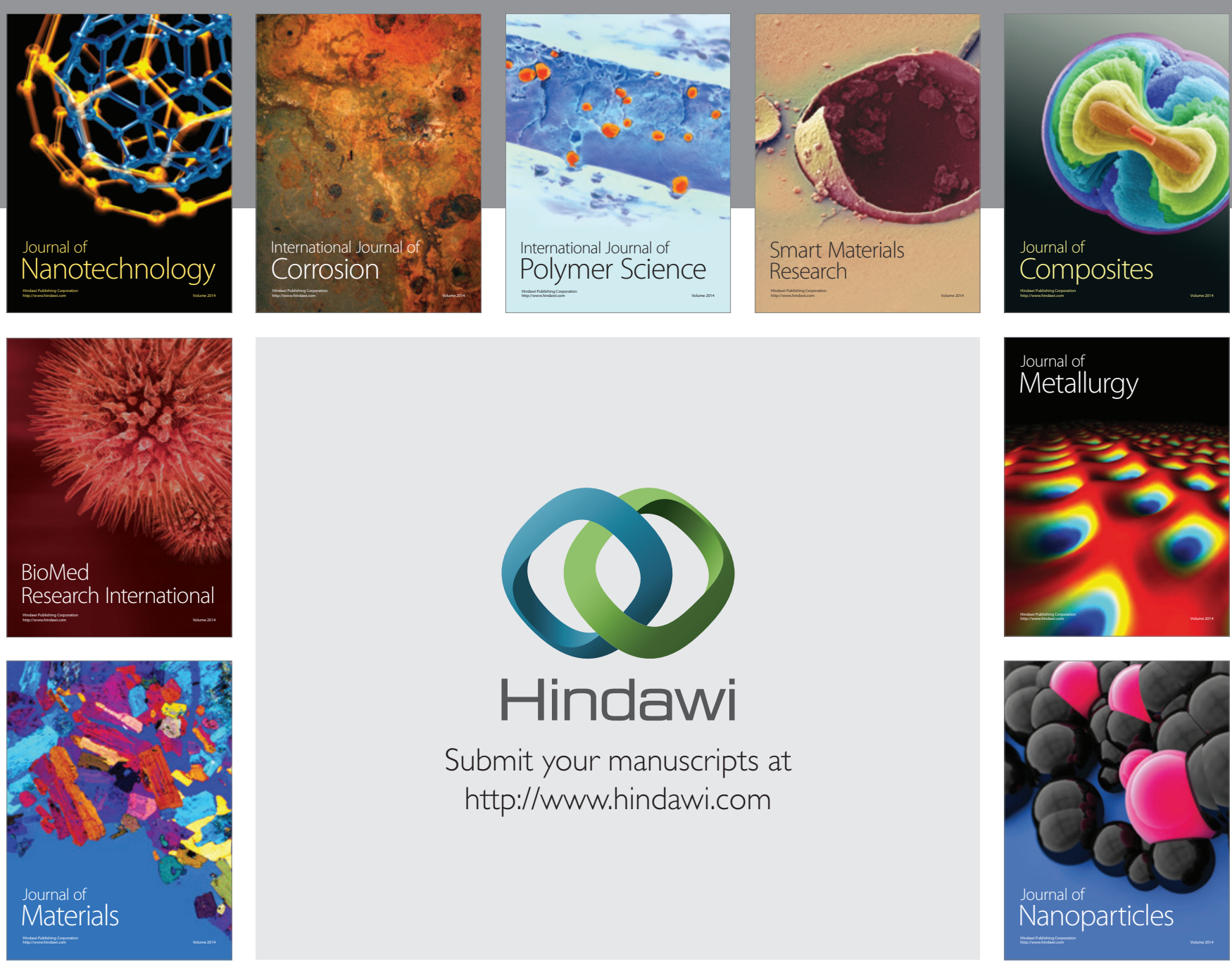

Submit your manuscripts at http://www.hindawi.com
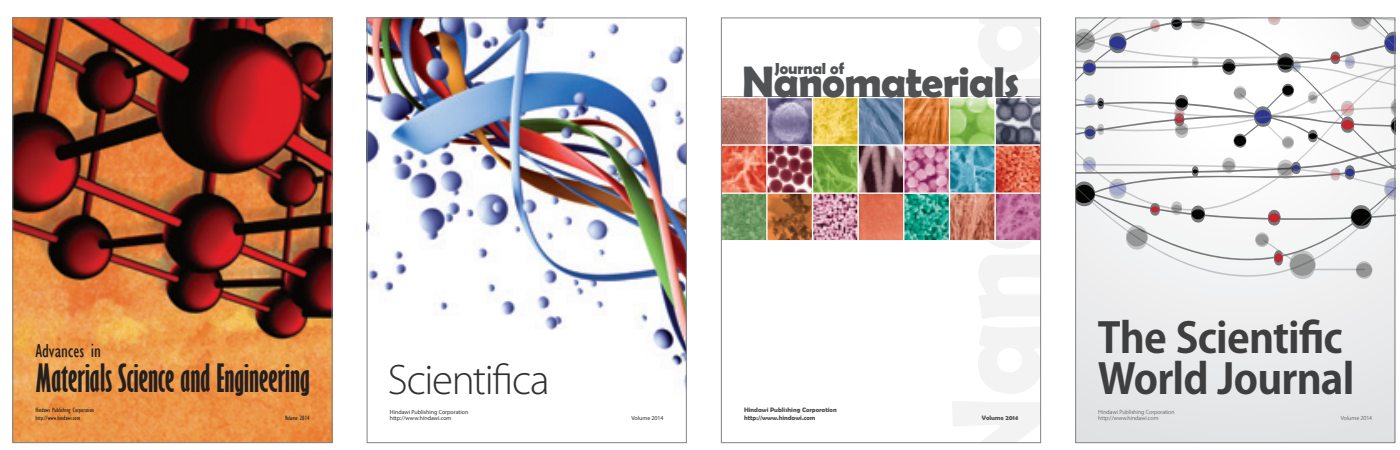

\section{The Scientific World Journal}
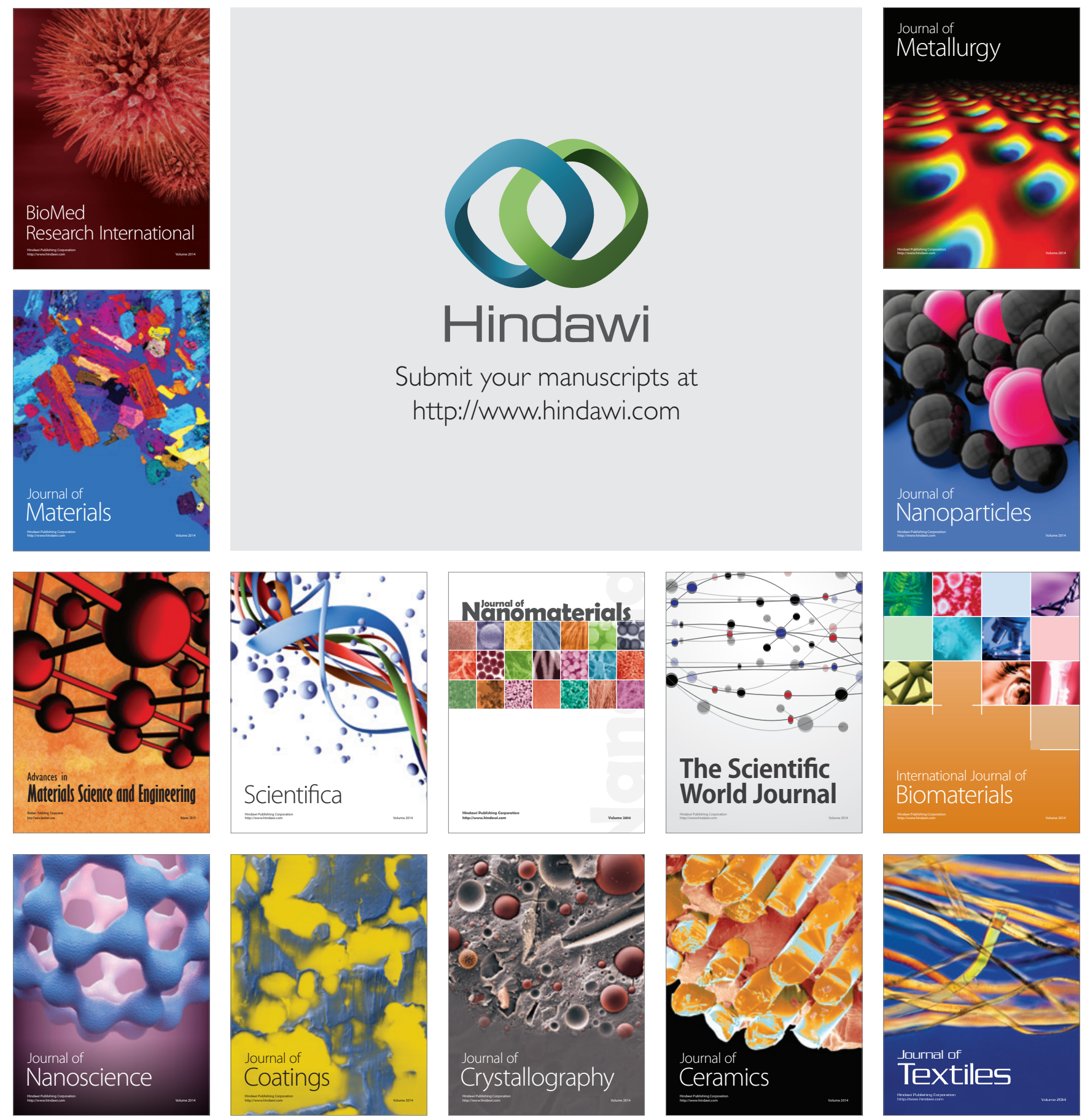